\title{
On the maximum average degree and the incidence chromatic number of a graph
}

\author{
Mohammad Hosseini Dolama ${ }^{1}$ and Eric Sopena ${ }^{2}$ \\ ${ }^{1}$ Department of Mathematics, Semnan University, Semnan, Iran \\ ${ }^{2}$ LaBRI, Université Bordeaux 1, 351 cours de la Libération, 33405 Talence Cedex, France
}

received Mar 2005, accepted Jul 2005.

\begin{abstract}
We prove that the incidence chromatic number of every 3-degenerated graph $G$ is at most $\Delta(G)+4$. It is known that the incidence chromatic number of every graph $G$ with maximum average degree $\operatorname{mad}(G)<3$ is at most $\Delta(G)+3$. We show that when $\Delta(G) \geq 5$, this bound may be decreased to $\Delta(G)+2$. Moreover, we show that for every graph $G$ with $\operatorname{mad}(G)<22 / 9$ (resp. with $\operatorname{mad}(G)<16 / 7$ and $\Delta(G) \geq 4$ ), this bound may be decreased to $\Delta(G)+2$ (resp. to $\Delta(G)+1$ ).
\end{abstract}

Keywords: incidence coloring, $k$-degenerated graph, planar graph, maximum average degree

\section{Introduction}

The concept of incidence coloring was introduced by Brualdi and Massey (3) in 1993.

Let $G=(V(G), E(G))$ be a graph. An incidence in $G$ is a pair $(v, e)$ with $v \in V(G), e \in E(G)$, such that $v$ and $e$ are incident. We denote by $I(G)$ the set of all incidences in $G$. For every vertex $v$, we denote by $I_{v}$ the set of incidences of the form $(v, v w)$ and by $A_{v}$ the set of incidences of the form $(w, w v)$. Two incidences $(v, e)$ and $(w, f)$ are adjacent if one of the following holds: $(i) v=w,(i i) e=f$ or $(i i i)$ the edge $v w$ equals $e$ or $f$.

A $k$-incidence coloring of a graph $G$ is a mapping $\sigma$ of $I(G)$ to a set $C$ of $k$ colors such that adjacent incidences are assigned distinct colors. The incidence chromatic number $\chi_{i}(G)$ of $G$ is the smallest $k$ such that $G$ admits a $k$-incidence coloring.

For a graph $G$, let $\Delta(G), \delta(G)$ denote the maximum and minimum degree of $G$ respectively. It is easy to observe that for every graph $G$ we have $\chi_{i}(G) \geq \Delta(G)+1$ (for a vertex $v$ of degree $\Delta(G)$ we must use $\Delta(G)$ colors for coloring $I_{v}$ and at least one additional color for coloring $A_{v}$ ). Brualdi and Massey proved the following upper bound:

Theorem 1 (3) For every graph $G, \chi_{i}(G) \leq 2 \Delta(G)$.

Guiduli (4) showed that the concept of incidence coloring is a particular case of directed star arboricity, introduced by Algor and Alon (1). Following an example from (1), Guiduli proved that there exist graphs $G$ with $\chi_{i}(G) \geq \Delta(G)+\Omega(\log \Delta(G))$. He also proved that For every graph $G, \chi_{i}(G) \leq \Delta(G)+$ $O(\log \Delta(G))$.

Concerning the incidence chromatic number of special classes of graphs, the following is known: 
- For every $n \geq 2, \chi_{i}\left(K_{n}\right)=n=\Delta\left(K_{n}\right)+1(\underline{3})$.

- For every $m \geq n \geq 2, \chi_{i}\left(K_{m, n}\right)=m+2=\Delta\left(K_{m, n}\right)+2$ (3).

- For every tree $T$ of order $n \geq 2, \chi_{i}(T)=\Delta(T)+1$ (3).

- For every Halin graph $G$ with $\Delta(G) \geq 5, \chi_{i}(G)=\Delta(G)+1$ ( 8 ).

- For every $k$-degenerated graph $G, \chi_{i}(G) \leq \Delta(G)+2 k-1$ (5).

- For every $K_{4}$-minor free graph $G, \chi_{i}(G) \leq \Delta(G)+2$ and this bound is tight (5).

- For every cubic graph $G, \chi_{i}(G) \leq 5$ and this bound is tight (6).

- For every planar graph $G, \chi_{i}(G) \leq \Delta(G)+7$ (5).

The maximum average degree of a graph $G$, denoted by $\operatorname{mad}(G)$, is defined as the maximum of the average degrees $a d(H)=2 \cdot|E(H)| /|V(H)|$ taken over all the subgraphs $H$ of $G$.

In this paper we consider the class of 3-degenerated graphs (recall that a graph $G$ is $k$-degenerated if $\delta(H) \leq k$ for every subgraph $H$ of $G$ ), which includes for instance the class of triangle-free planar graphs and the class of graphs with maximum average degree at most 3 . More precisely, we shall prove the following:

1. If $G$ is a 3-degenerated graph, then $\chi_{i}(G) \leq \Delta(G)+4$ (Theorem 2).

2. If $G$ is a graph with $\operatorname{mad}(G)<3$, then $\chi_{i}(G) \leq \Delta(G)+3$ (Corollary 5 .

3. If $G$ a graph with $\operatorname{mad}(G)<3$ and $\Delta(G) \geq 5$, then $\chi_{i}(G) \leq \Delta(G)+2$ (Theorem 8 .

4. If $G$ is a graph with $\operatorname{mad}(G)<22 / 9$, then $\chi_{i}(G) \leq \Delta(G)+2$ (Theorem 11 .

5. If $G$ is a graph with $\operatorname{mad}(G)<16 / 7$ and $\Delta(G) \geq 4$, then $\chi_{i}(G)=\Delta(G)+1$ (Theorem 13).

In fact we shall prove something stronger, namely that one can construct for these classes of graphs incidence colorings such that for every vertex $v$, the number of colors that are used on the incidences of the form $(w, w v)$ is bounded by some fixed constant not depending on the maximum degree of the graph.

More precisely, we define a $(k, \ell)$-incidence coloring of a graph $G$ as a $k$-incidence coloring $\sigma$ of $G$ such that for every vertex $v \in V(G),\left|\sigma\left(A_{v}\right)\right| \leq \ell$.

We end this section by introducing some notation that we shall use in the rest of the paper.

Let $G$ be a graph. If $v$ is a vertex in $G$ and $v w$ is an edge in $G$, we denote by $N_{G}(v)$ the set of neighbors of $v$, by $d_{G}(v)=\left|N_{G}(v)\right|$ the degree of $v$, by $G \backslash v$ the graph obtained from $G$ by deleting the vertex $v$ and by $G \backslash v w$ the graph obtained from $G$ by deleting the edge $v w$.

Let $G$ be a graph and $\sigma^{\prime}$ a partial incidence coloring of $G$, that is an incidence coloring only defined on some subset $I$ of $I(G)$. For every uncolored incidence $(v, v w) \in I(G) \backslash I$, we denote by $F_{G}^{\sigma^{\prime}}(v, v w)$ the set of forbidden colors of $(v, v w)$, that is:

$$
F_{G}^{\sigma^{\prime}}(v, v w)=\sigma^{\prime}\left(A_{v}\right) \cup \sigma^{\prime}\left(I_{v}\right) \cup \sigma^{\prime}\left(I_{w}\right) .
$$




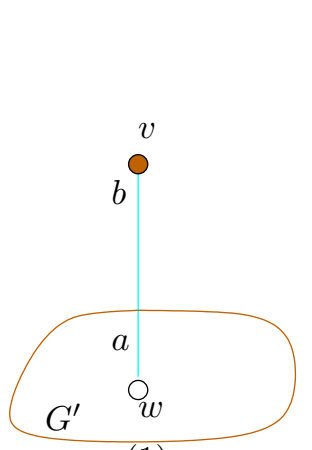

(1)

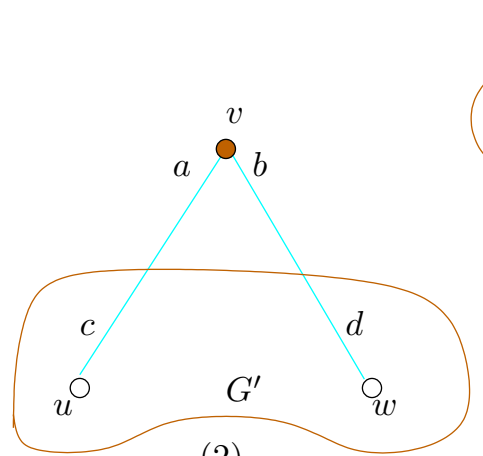

(2)

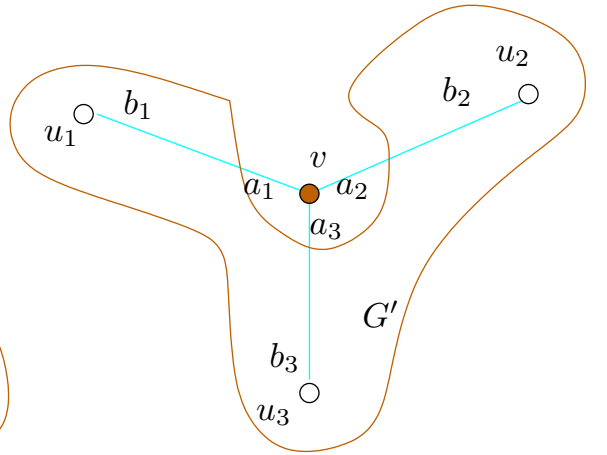

(3)

Fig. 1: Configurations for the proof of Theorem 2

We shall often say that we extend such a partial incidence coloring $\sigma^{\prime}$ to some incidence coloring $\sigma$ of $G$. In that case, it should be understood that we set $\sigma(v, v w)=\sigma^{\prime}(v, v w)$ for every incidence $(v, v w) \in I$.

We shall make extensive use of the fact that every $(k, \ell)$-incidence coloring may be viewed as a $\left(k^{\prime}, \ell\right)$ incidence coloring for any $k^{\prime}>k$.

Drawing convention. In a figure representing a forbidden configuration, all the neighbors of "black" or "grey" vertices are drawn, whereas "white" vertices may have other neighbors in the graph.

\section{3-degenerated graphs}

In this section, we prove the following:

Theorem 2 Every 3-degenerated graph $G$ admits a $(\Delta(G)+4,3)$-incidence coloring. Therefore, $\chi_{i}(G) \leq$ $\Delta(G)+4$.

Proof: Let $G$ be a 3-degenerated graph. Observe first that if $\Delta(G) \leq 3$ then, by Theorem $1, \chi_{i}(G) \leq$ $2 \Delta(G)<\Delta(G)+4 \leq 7$ and every $(\Delta(G)+4)$-incidence coloring of $G$ is obviously a $(\Delta(G)+4,3)$ incidence coloring.

Therefore, we assume $\Delta(G) \geq 4$ and we prove the theorem by induction on the number of vertices of $G$. If $G$ has at most 5 vertices then $G \subseteq K_{5}$. Since for every $k>0, \chi_{i}\left(K_{n}\right)=n$, we obtain $\chi_{i}(G) \leq \chi_{i}\left(K_{5}\right)=\Delta\left(K_{5}\right)+1=5$, and every 5 -incidence coloring of $G$ is obviously a $(\Delta(G)+4,3)$ incidence coloring. We assume now that $G$ has $n+1$ vertices, $n \geq 5$, and that the theorem is true for all 3-degenerated graphs with at most $n$ vertices.

Let $v$ be a vertex of $G$ with minimum degree. Since $G$ is 3-degenerated, we have $d_{G}(v) \leq 3$. We consider three cases according to $d_{G}(v)$.

$d_{G}(v)=1:$

Let $w$ denote the unique neighbor of $v$ in $G$ (see Figure 1 (1)). Due to the induction hypothesis, the graph $G^{\prime}=G \backslash v$ admits a $(\Delta(G)+4,3)$-incidence coloring $\sigma^{\prime}$. We extend $\sigma^{\prime}$ to a $(\Delta(G)+4,3)$ incidence coloring of $G$. Since $\left|F_{G}^{\sigma^{\prime}}(w, w v)\right|=\left|\sigma^{\prime}\left(I_{w}\right) \cup \sigma^{\prime}\left(A_{w}\right)\right| \leq \Delta(G)-1+3=\Delta(G)+2$, 
there is a color $a$ such that $a \notin F_{G}^{\sigma^{\prime}}(w, w v)$. We then set $\sigma(w, w v)=a$ and $\sigma(v, v w)=b$, for any color $b$ in $\sigma^{\prime}\left(A_{w}\right)$.

$d_{G}(v)=2:$

Let $u, w$ be the two neighbors of $v$ in $G$ (see Figure 1 (2)). Due to the induction hypothesis, the graph $G^{\prime}=G \backslash v$ admits a $(\Delta(G)+4,3)$-incidence coloring $\sigma^{\prime}$. We extend $\sigma^{\prime}$ to a $(\Delta(G)+4,3)$ incidence coloring $\sigma$ of $G$ as follows. We first set $\sigma(v, v u)=a$ for a color $a \in \sigma\left(A_{u}\right)$ (if $d_{G}(u)=$ 1 , we have the case 1). Now, if $\left|\sigma^{\prime}\left(A_{w}\right)\right| \geq 2$, there is a color $b \in \sigma^{\prime}\left(A_{w}\right) \backslash\{a\}$ and if $\left|\sigma^{\prime}\left(A_{w}\right)\right|=1$, since $\left|F_{G}^{\sigma}(v, v w)\right|=\left|\sigma^{\prime}\left(I_{w}\right) \cup\{a\}\right| \leq \Delta(G)-1+1=\Delta(G)$, there is a color $b$ distinct from $a$ such that $b \notin F_{G}^{\sigma}(v, v w)$. We set $\sigma(v, v w)=b$.

We still have to color the two incidences $(u, u v)$ and $(w, w v)$. Since $a \in \sigma^{\prime}\left(A_{u}\right)$, we have $\left|F_{G}^{\sigma}(u, u v)\right|=\left|\sigma^{\prime}\left(I_{u}\right) \cup \sigma^{\prime}\left(A_{u}\right) \cup\{a, b\}\right| \leq \Delta(G)-1+3+2-1=\Delta(G)+3$. Therefore, there is a color $c$ such that $c \notin F_{G}^{\sigma}(u, u v)$. Similarly, since $b \in \sigma\left(A_{w}\right)$, we have $\left|F_{G}^{\sigma}(w, w v)\right| \leq \Delta(G)+3$ and there exists a color $d$ such that $d \notin F_{G}^{\sigma}(w, w v)$. We can extend $\sigma^{\prime}$ to a $(\Delta(G)+4,3)$-incidence coloring $\sigma$ of $G$ by setting $\sigma(u, u v)=c$ and $\sigma(w, w v)=d$.

$d_{G}(v)=3:$

Let $u_{1}, u_{2}$ and $u_{3}$ be the three neighbors of $v$ in $G$ (see Figure 1 (3)). Due to the induction hypothesis, the graph $G^{\prime}=G \backslash v$ admits a $(\Delta(G)+4,3)$-incidence coloring $\sigma^{\prime}$.

Observe first that for every $i, 1 \leq i \leq 3$, since $\left|F_{G}^{\sigma^{\prime}}\left(v, v u_{i}\right)\right| \leq \Delta(G)-1$ and since we have $\Delta(G)+4$ colors, we have at least five colors which are not in $F_{G}^{\sigma^{\prime}}\left(v, v u_{i}\right)$. Moreover, if $\left|A_{u_{i}}\right|<3$ then any of these five colors may be assigned to the incidence $\left(v, v u_{i}\right)$ whereas we have only three possible choices (among these five) if $\left|A_{u_{i}}\right|=3$. In the following, we shall see that having only three available colors is enough, and therefore assume that $\left|\sigma^{\prime}\left(A_{u_{i}}\right)\right|=3$ for every $i, 1 \leq i \leq 3$.

We define the sets $B$ and $B_{i, j}$ as follows:

- $\forall i, j, 1 \leq i, j \leq 3, i \neq j, B_{i, j}:=\left(\sigma^{\prime}\left(I_{u_{i}}\right) \cup \sigma^{\prime}\left(A_{u_{i}}\right)\right) \cap \sigma^{\prime}\left(A_{u_{j}}\right)$

- $B:=\bigcup_{1 \leq i, j \leq 3} B_{i, j}, i \neq j$.

We consider now four subcases according to the degrees of $u_{1}, u_{2}$ and $u_{3}$ :

1. $\forall i, 1 \leq i \leq 3, d_{G}\left(u_{i}\right)<\Delta(G)$.

In this case, since we have 3 colors for the incidence $\left(v, v u_{i}\right)$ for every $i, 1 \leq i \leq 3$, we can find 3 distinct colors $a_{1}, a_{2}, a_{3}$ such that $a_{i} \notin F_{G}^{\sigma^{\prime}}\left(v, v u_{i}\right)$. We set $\sigma\left(v, v u_{i}\right)=a_{i}$ for every $i$, $1 \leq i \leq 3$.

We still have to color the three incidences $\left(u_{i}, u_{i} v\right), 1 \leq i \leq 3$. Since $a_{i} \in \sigma\left(A_{u_{i}}\right)$, we have $\left|F_{G}^{\sigma}\left(u_{i}, u_{i} v\right)\right|=\left|\sigma\left(I_{u_{i}}\right) \cup \sigma\left(A_{u_{i}}\right) \cup\left\{a_{1}, a_{2}, a_{3}\right\}\right| \leq \Delta(G)-2+3+3-1=\Delta(G)+3$ for every $i, 1 \leq i \leq 3$. So, there exist three colors $b_{1}, b_{2}, b_{3}$ such that $b_{i} \notin F_{G}^{\sigma}\left(u_{i}, u_{i} v\right), 1 \leq i \leq 3$. We can extend $\sigma^{\prime}$ to a $(\Delta(G)+4,3)$-incidence coloring $\sigma$ of $G$ by setting $\sigma\left(u_{i}, u_{i} v\right)=b_{i}$ for every $i, 1 \leq i \leq 3$.

2. Only one of the vertices $u_{i}$ is of degree $\Delta(G)$.

We can suppose without loss of generality that $d_{G}\left(u_{1}\right), d_{G}\left(u_{2}\right)<\Delta(G)$ and $d_{G}\left(u_{3}\right)=$ $\Delta(G)$. 
Since $\left|\sigma^{\prime}\left(I_{u_{3}}\right) \cup \sigma^{\prime}\left(A_{u_{3}}\right)\right|=\Delta(G)-1+3=\Delta(G)+2$ and $\left|\sigma^{\prime}\left(A_{u_{1}}\right)\right|=3$, we have $B_{3,1} \neq \emptyset$. Let $a_{1} \in B_{3,1}$. Since $\left|\sigma^{\prime}\left(A_{u_{i}}\right)\right|=3$ for every $i, 1 \leq i \leq 3$, there exist two distinct colors $a_{2}$ and $a_{3}$ distinct from $a_{1}$ such that $a_{2} \in \sigma^{\prime}\left(A_{u_{2}}\right)$ and $a_{3} \in \sigma^{\prime}\left(A_{u_{3}}\right)$. We set $\sigma\left(v, v u_{i}\right)=a_{i}$ for every $i, 1 \leq i \leq 3$.

We still have to color the three incidences of form $\left(u_{i}, u_{i} v\right)$. Since $a_{1} \in B_{3,1}$ and $a_{3} \in$ $\sigma^{\prime}\left(A_{u_{3}}\right)$ we have:

$$
\begin{gathered}
\left|F_{G}^{\sigma}\left(u_{3}, u_{3} v\right)\right|=\left|\sigma^{\prime}\left(I_{u_{3}}\right) \cup \sigma\left(A_{u_{3}}\right) \cup\left\{a_{1}, a_{2}, a_{3}\right\}\right| \\
\leq \Delta(G)-1+3+3-1-1=\Delta+3
\end{gathered}
$$

and since $a_{i} \in \sigma^{\prime}\left(A_{u_{i}}\right)$ for every $i=1,2$ we have:

$$
\begin{gathered}
\left|F_{G}^{\sigma^{\prime}}\left(u_{i}, u_{i} v\right)\right|=\left|\sigma^{\prime}\left(I_{u_{i}}\right) \cup \sigma^{\prime}\left(A_{u_{i}}\right) \cup\left\{a_{1}, a_{2}, a_{3}\right\}\right| \\
\leq \Delta(G)-2+3+3-1=\Delta+3 .
\end{gathered}
$$

Therefore, there exist three colors $b_{1}, b_{2}, b_{3}$ such that $b_{i} \notin F_{G}^{\sigma}\left(u_{i}, u_{i} v\right) \cup\left\{a_{1}, a_{2}, a_{3}\right\}, 1 \leq i \leq$ 3. We can extend $\sigma^{\prime}$ to a $(\Delta(G)+4,3)$-incidence coloring $\sigma$ of $G$ by setting $\sigma\left(u_{i}, u_{i} v\right)=b_{i}$ for every $i, 1 \leq i \leq 3$.

3. Only one vertex among the $u_{i}$ 's is of degree less than $\Delta(G)$.

We can suppose without loss of generality that $d_{G}\left(u_{1}\right)<\Delta(G)$ and $d_{G}\left(u_{2}\right)=d_{G}\left(u_{3}\right)=$ $\Delta(G)$.

Similarly to the previous case, we have $B_{2,1} \neq \emptyset$ and $B_{3,2} \neq \emptyset$. We consider two cases:

$B_{2,1} \neq B_{3,2}$

Let $a_{1} \in B_{2,1}, a_{2} \in B_{3,2} \backslash\left\{a_{1}\right\}$ and $a_{3} \in \sigma^{\prime}\left(A_{u_{3}}\right) \backslash\left\{a_{1}, a_{2}\right\}$. We set $\sigma\left(v, v u_{i}\right)=a_{i}$ for every $i, 1 \leq i \leq 3$.

We still have to color the three incidences $\left(u_{i}, u_{i} v\right), 1 \leq i \leq 3$. Since $a_{1} \in \sigma^{\prime}\left(A_{u_{1}}\right)$ we have:

$$
\begin{gathered}
\left|F_{G}^{\sigma}\left(u_{1}, u_{1} v\right)\right|=\left|\sigma^{\prime}\left(I_{u_{1}}\right) \cup \sigma\left(A_{u_{1}}\right) \cup\left\{a_{1}, a_{2}, a_{3}\right\}\right| \\
\leq \Delta(G)-2+3+3-1=\Delta(G)+3
\end{gathered}
$$

and since $a_{i} \in B_{i+1, i}$ for $i=1,2$ and $a_{j} \in \sigma^{\prime}\left(A_{u_{j}}\right)$ for $j=2,3$, we have:

$$
\begin{gathered}
\left|F_{G}^{\sigma}\left(u_{i}, u_{i} v\right)\right|=\left|\sigma^{\prime}\left(I_{u_{i}}\right) \cup \sigma\left(A_{u_{i}}\right) \cup\left\{a_{1}, a_{2}, a_{3}\right\}\right| \\
\leq \Delta(G)-1+3+3-1-1=\Delta(G)+3 .
\end{gathered}
$$

Therefore, there exist three colors $b_{1}, b_{2}, b_{3}$ such that $b_{i} \notin F_{G}^{\sigma}\left(u_{i}, u_{i} v\right), 1 \leq i \leq 3$. We can extend $\sigma^{\prime}$ to a $(\Delta(G)+4,3)$-incidence coloring $\sigma$ of $G$ by setting $\sigma\left(u_{i}, u_{i} v\right)=b_{i}$ for every $i, 1 \leq i \leq 3$.

$B_{2,1}=B_{3,2}$

Let $a_{1} \in B_{2,1}=B_{3,2}, a_{2} \in \sigma^{\prime}\left(A_{u_{2}}\right) \backslash\left\{a_{1}\right\}$ and $a_{3} \in \sigma^{\prime}\left(A_{u_{3}}\right) \backslash\left\{a_{1}, a_{2}\right\}$. We set $\sigma\left(v, v u_{i}\right)=a_{i}$ for every $i, 1 \leq i \leq 3$. 
We still have to color the three incidences $\left(u_{i}, u_{i} v\right), 1 \leq i \leq 3$. Since $a_{1} \in \sigma^{\prime}\left(A_{u_{1}}\right)$ we have:

$$
\begin{gathered}
\left|F_{G}^{\sigma}\left(u_{1}, u_{1} v\right)\right|=\left|\sigma^{\prime}\left(I_{u_{1}}\right) \cup \sigma\left(A_{u_{1}}\right) \cup\left\{a_{1}, a_{2}, a_{3}\right\}\right| \\
\leq \Delta(G)-2+3+3-1=\Delta(G)+3
\end{gathered}
$$

and since $a_{1} \in B_{2,1}=B_{3,2}$ and $a_{j} \in \sigma^{\prime}\left(A_{u_{j}}\right)$ for $j=2,3$, we have:

$$
\begin{gathered}
\left|F_{G}^{\sigma}\left(u_{i}, u_{i} v\right)\right|=\left|\sigma^{\prime}\left(I_{u_{i}}\right) \cup \sigma\left(A_{u_{i}}\right) \cup\left\{a_{1}, a_{2}, a_{3}\right\}\right| \\
\leq \Delta(G)-1+3+3-1-1=\Delta(G)+3 .
\end{gathered}
$$

Therefore, there exist three colors $b_{1}, b_{2}, b_{3}$ such that $b_{i} \notin F_{G}^{\sigma}\left(u_{i}, u_{i} v\right), 1 \leq i \leq 3$. We can extend $\sigma^{\prime}$ to a $(\Delta(G)+4,3)$-incidence coloring $\sigma$ of $G$ by setting $\sigma\left(u_{i}, u_{i} v\right)=b_{i}$ for every $i, 1 \leq i \leq 3$.

4. $d_{G}\left(u_{1}\right)=d_{G}\left(u_{2}\right)=d_{G}\left(u_{3}\right)=\Delta(G)$.

Similarly to the case $(b)$ we have $B_{i, j} \neq \emptyset$ for every $i$ and $j, 1 \leq i, j \leq 3$ and thus $|B| \geq 1$. We prove first that in this case $|B| \geq 2$. Suppose that $|B|=|\{x\}|=1$; in other words, $\left(\left(\sigma^{\prime}\left(I_{u_{i}}\right) \cup A_{u_{i}}^{\prime}\right) \cap A_{u_{j}}^{\prime}\right)=\{x\}$ for every $i$ and $j, 1 \leq i, j \leq 3$. Thus we have:

$$
\begin{aligned}
\mid \sigma^{\prime}\left(A_{u_{1}}\right) \cup \sigma^{\prime}\left(I_{u_{1}}\right) \cup \sigma^{\prime}\left(A_{u_{2}}\right) & \cup \sigma^{\prime}\left(A_{u_{3}}\right) \mid=\Delta(G)-1+3+3+3-1-1 \\
& =\Delta(G)+6 .
\end{aligned}
$$

But the relation $(1)$ is in contradiction with the fact that $\sigma^{\prime}$ is a $(\Delta(G)+4,3)$-incidence coloring and we then get $|B| \geq 2$.

Let $a_{1}$ and $a_{2}$ be two distinct colors in $B$. We can suppose without loss of generality that $a_{1} \in B_{2,1}$ and $a_{2} \in B_{3,2}$.

We consider the two following subcases:

$B_{1,3} \backslash\left\{a_{1}, a_{2}\right\} \neq \emptyset$

Let $a_{3}$ be a color in $B_{1,3} \backslash\left\{a_{1}, a_{2}\right\}$. We set $\sigma\left(v, v u_{i}\right)=a_{i}$ for every $i, 1 \leq i \leq 3$.

Since $a_{i} \in B_{j, i}=\left(\sigma^{\prime}\left(I_{u_{j}}\right) \cup \sigma^{\prime}\left(A_{u_{j}}\right)\right) \cap \sigma^{\prime}\left(A_{u_{i}}\right), j=i+1 \bmod 3$, and $a_{i} \in \sigma^{\prime}\left(A_{u_{i}}\right)$ for every $i, 1 \leq i \leq 3$, we have:

$$
\begin{gathered}
\left|F_{G}^{\sigma}\left(u_{i}, u_{i} v\right)\right|=\left|\sigma^{\prime}\left(I_{u_{i}}\right) \cup \sigma^{\prime}\left(A_{u_{i}}\right) \cup\left\{a_{1}, a_{2}, a_{3}\right\}\right| \\
\leq \Delta(G)-1+3+3-1-1=\Delta(G)+3 .
\end{gathered}
$$

Therefore, there exist three colors $b_{1}, b_{2}, b_{3}$ such that $b_{i} \notin F_{G}^{\sigma}\left(u_{i}, u_{i} v\right), 1 \leq i \leq 3$. We can extend $\sigma^{\prime}$ to a $(\Delta(G)+4,3)$-incidence coloring $\sigma$ of $G$ by setting $\sigma\left(u_{i}, u_{i} v\right)=b_{i}$ for every $i, 1 \leq i \leq 3$.

$B_{1,3} \backslash\left\{a_{1}, a_{2}\right\}=\emptyset$

Since $B_{1,3} \neq \emptyset$ we can suppose without loss of generality that $a_{2} \in B_{1,3}$. Let $a_{3} \in$ $\sigma^{\prime}\left(A_{u_{3}}\right) \backslash\left\{a_{1}, a_{2}\right\}$. We set $\sigma\left(v, v u_{i}\right)=a_{i}$ for every $i, 1 \leq i \leq 3$.

Since $a_{i} \in B_{j, i}=\left(\sigma^{\prime}\left(I_{u_{j}}\right) \cup \sigma^{\prime}\left(A_{u_{j}}\right)\right) \cap \sigma^{\prime}\left(A_{u_{i}}\right), j=i+1 \bmod 3$, and $a_{i} \in \sigma^{\prime}\left(A_{u_{i}}\right)$ for $i=1,2$, we have:

$$
\left|F_{G}^{\sigma}\left(u_{i}, u_{i} v\right)\right|=\left|\sigma^{\prime}\left(I_{u_{i}}\right) \cup \sigma^{\prime}\left(A_{u_{i}}\right) \cup\left\{a_{1}, a_{2}, a_{3}\right\}\right|
$$




$$
\leq \Delta(G)-1+3+3-1-1=\Delta(G)+3
$$

and since $a_{2} \in \sigma^{\prime}\left(I_{u_{1}}\right) \cup \sigma^{\prime}\left(A_{u_{1}}\right)$ and $a_{1} \in \sigma^{\prime}\left(A-u_{1}\right)$ we have:

$$
\begin{gathered}
\left|F_{G}^{\sigma}\left(u_{1}, u_{1} v\right)\right|=\left|\sigma^{\prime}\left(I_{u_{1}}\right) \cup \sigma^{\prime}\left(A_{u_{1}}\right) \cup\left\{a_{1}, a_{2}, a_{3}\right\}\right| \\
\quad \leq \Delta(G)-1+3+3-1-1=\Delta(G)+3 .
\end{gathered}
$$

Therefore, there exist three colors $b_{1}, b_{2}, b_{3}$ such that $b_{i} \notin F_{G}^{\sigma}\left(u_{i}, u_{i} v\right), 1 \leq i \leq 3$. We can extend $\sigma^{\prime}$ to a $(\Delta(G)+4,3)$-incidence coloring $\sigma$ of $G$ by setting $\sigma\left(u_{i}, u_{i} v\right)=b_{i}$ for every $i, 1 \leq i \leq 3$.

It is easy to check that in all cases we obtain a $(\Delta(G)+4,3)$-incidence coloring of $G$ and the theorem is proved.

Since every triangle free planar graph is 3-degenerated, we have:

Corollary 3 For every triangle free planar graph $G, \chi_{i}(G) \leq \Delta(G)+4$.

\section{Graphs with bounded maximum average degree}

In this section we study the incidence chromatic number of graphs with bounded maximum average degree. The following result has been proved in (5).

Theorem 4 Every $k$-degenerated graph $G$ admits a $(\Delta(G)+2 k-1, k)$-incidence coloring.

Since every graph $G$ with $\operatorname{mad}(G)<3$ is 2-degenerated, we get the following:

Corollary 5 Every graph $G$ with $\operatorname{mad}(G)<3$ admits a $(\Delta(G)+3,2)$-incidence coloring. Therefore, $\chi_{i}(G) \leq \Delta(G)+3$.

Concerning planar graphs, we have the following:

Observation 6 (2) For every planar graph $G$ with girth at least $g, \operatorname{mad}(G)<2 g /(g-2)$.

Hence, we obtain:

Corollary 7 Every planar graph $G$ with girth $g \geq 6$ admits $a(\Delta(G)+3,2)$-incidence coloring. Therefore, $\chi_{i}(G) \leq \Delta(G)+3$.

Proof: By Observation 6 we have $\operatorname{mad}(G)<2 g /(g-2) \leq(2 \times 6) /(6-2)=3$ and we get the result from Corollary 5

If the graph has maximum degree at least 5, the previous result can be improved:

Theorem 8 Every graph $G$ with $\operatorname{mad}(G)<3$ and $\Delta(G) \geq 5$ admits $a(\Delta(G)+2,2)$-incidence coloring. Therefore, $\chi_{i}(G) \leq \Delta(G)+2$.

Proof: Suppose that the theorem is false and let $G$ be a minimal counter-example (with respect to the number of vertices). We first show that $G$ must avoid all the configurations depicted in Fig. 2 . 


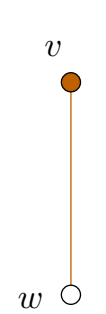

(1) $d_{G}(v)=1$

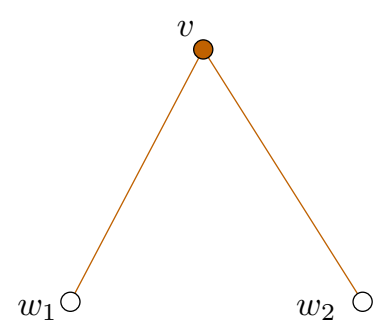

(2) $d_{G}\left(w_{2}\right)<5$

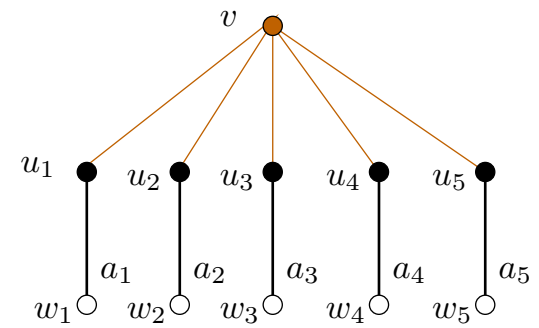

(3) $d_{G}(v)=5$

Fig. 2: Forbidden configurations for the proof of Theorem 8

(1) Let $w$ denote the unique neighbor of $v$ in $G$. Due to the minimality of $G$, the graph $G^{\prime}=G \backslash v$ admits a $(\Delta(G)+2,2)$-incidence coloring $\sigma^{\prime}$. We extend $\sigma^{\prime}$ to a $(\Delta(G)+2,2)$-incidence coloring $\sigma$ of $G$. Since $\left|F_{G}^{\sigma}(w, w v)\right|=\left|\sigma^{\prime}\left(I_{w}\right) \cup \sigma^{\prime}\left(A_{w}\right)\right| \leq \Delta(G)-1+2=\Delta(G)+1$, there is a color $a$ such that $a \notin F_{G}^{\sigma}(w, w v)$. We set $\sigma(w, w v)=a$ and $\sigma(v, v w)=b$, for any color $b$ in $\sigma^{\prime}\left(A_{w}\right)$.

(2) Let $w_{1}, w_{2}$ denote the two neighbors of $v$ in $G$. Due to the minimality of $G$, the graph $G^{\prime}=G \backslash v$ admits a $(\Delta(G)+2,2)$-incidence coloring $\sigma^{\prime}$. We extend $\sigma^{\prime}$ to a $(\Delta(G)+2,2)$-incidence coloring $\sigma$ of $G$.

Since $\left|F_{G}^{\sigma}\left(w_{1}, w_{1} v\right)\right|=\left|\sigma^{\prime}\left(I_{w_{1}}\right) \cup \sigma^{\prime}\left(A_{w_{1}}\right)\right| \leq \Delta(G)-1+2=\Delta(G)+1$ and since we have $\Delta(G)+2$ possible colors, there is a color $a$ such that $a \notin F_{G}^{\sigma}\left(w_{1}, w_{1} v\right)$. We set $\sigma\left(w_{1}, w_{1} v\right)=a$. If $\left|\sigma^{\prime}\left(A_{w_{2}}\right) \backslash\{a\}\right| \geq 1$ then there is a color $b \in \sigma^{\prime}\left(A_{w_{2}}\right) \backslash\{a\}$ and if $\sigma^{\prime}\left(A_{w_{2}}\right)=\{a\}$, since $\left|F_{G}^{\sigma}\left(v, v w_{2}\right)\right|=\left|\sigma^{\prime}\left(I_{w_{2}}\right) \cup\{a\}\right| \leq 3+1=4 \leq \Delta(G)-1$, there is a color $b$ such that $b \notin F_{G}^{\sigma}\left(v, v w_{2}\right)$. We set $\sigma\left(v, v w_{2}\right)=b$.

Now, if $\left|\sigma^{\prime}\left(A_{w_{1}}\right) \backslash\{b\}\right| \geq 1$ then there is a color $c \in \sigma^{\prime}\left(A_{w_{1}}\right) \backslash\{b\}$ and if $\sigma^{\prime}\left(A_{w_{1}}\right)=\{b\}$, since $\left|F_{G}^{\sigma}\left(v, v w_{1}\right)\right|=\left|\sigma\left(I_{w_{1}}\right) \cup\{b\}\right| \leq \Delta(G)+1$, there is a color $c$ such that $c \notin F_{G}^{\sigma}\left(v, v w_{1}\right)$. We set $\sigma\left(v, v w_{1}\right)=c$.

Since $\left|F_{G}^{\sigma}\left(w_{2}, w_{2} v\right)\right|=\left|\sigma^{\prime}\left(I_{w_{2}}\right) \cup \sigma\left(A_{w_{2}}\right) \cup\{c\}\right| \leq 3+2+1=6 \leq \Delta(G)+1$, there is a color $d$ such that $d \notin F_{G}^{\sigma}\left(w_{2}, w_{2} v\right)$ and we set $\sigma\left(w_{2}, w_{2} v\right)=d$.

(3) Let $u_{i}, 1 \leq i \leq 5$, denote the five neighbors of $v$ and $w_{i}$ denote the other neighbor of $u_{i}$ in $G$ (see Figure 2.(3)). Due to the minimality of $G$, the graph $G^{\prime}=G \backslash v$ admits a $(\Delta(G)+2,2)$-incidence coloring $\sigma^{\prime}$. We extend $\sigma^{\prime}$ to a $(\Delta(G)+2,2)$-incidence coloring $\sigma$ of $G$.

Let $a_{i}=\sigma^{\prime}\left(w_{i}, w_{i} u_{i}\right), 1 \leq i \leq 5$. Since we have $\Delta(G)+2 \geq 7$ colors, there is a color $x$ distinct from $a_{i}$ for every $i, 1 \leq i \leq 5$.

Since $\left|F_{G}^{\sigma^{\prime}}\left(u_{i}, u_{i} w_{i}\right)\right|=\left|\sigma^{\prime}\left(I_{w_{i}}\right)\right| \leq \Delta(G)$ we have two possible colors for the incidence $\left(u_{i}, u_{i} w_{i}\right)$ for every $i, 1 \leq i \leq 5$. So, we can suppose that $\sigma^{\prime}\left(u_{i}, u_{i} w_{i}\right) \neq x$ for every $i, 1 \leq i \leq 5$. We set $\sigma\left(u_{i}, u_{i} v\right)=x$ for every $i, 1 \leq i \leq 5$.

Since $F_{G}^{\sigma}\left(v, v u_{i}\right)=\left\{x, \sigma^{\prime}\left(u_{i}, u_{i} w_{i}\right)\right\}$ for every $i, 1 \leq i \leq 5$, and since we have at least 7 colors, there is 5 distinct colors $c_{1}, c_{2}, \ldots, c_{5}$ such that $c_{i} \notin\left\{x, \sigma^{\prime}\left(u_{i}, u_{i} w_{i}\right)\right\}, 1 \leq i \leq 5$, and we set $\sigma\left(v, v u_{i}\right)=c_{i}$ for every $i, 1 \leq i \leq 5$. 
It is easy to check that in every case we have obtained a $(\Delta(G)+2,2)$-incidence coloring of $G$, which contradicts our assumption.

We now associate with each vertex $v$ of $G$ an initial charge $d(v)=d_{G}(v)$, and we use the following discharging procedure: each vertex of degree at least 5 gives $1 / 2$ to each of its 2 -neighbors.

We shall prove that the modernized degree $d^{*}$ of each vertex of $G$ is at least 3 which contradicts the assumption $\operatorname{mad}(G)<3\left(\right.$ since $\sum_{u \in G} d^{*}(u)=\sum_{u \in G} d(u)$ ). Let $v$ be a vertex of $G$; we consider the possible cases for old degree $d_{G}(v)$ of $v$ (since $G$ does not contain the configuration 2(1), we have $d_{G}(v) \geq 2$ ):

$d_{G}(v)=2$.

Since $G$ does not contain the configuration 2(2) the two neighbors of $v$ are of degree at least 5 . Therefore, $v$ receives $1 / 2$ from each of its neighbors so that $d^{*}(v)=2+1 / 2+1 / 2=3$.

$3 \leq d_{G}(v) \leq 4$

In this case we have $d^{*}(v)=d_{G}(v) \geq 3$.

$d_{G}(v)=5$.

Since $G$ does not contain the configuration $2(3)$ at least one of the neighbors of $v$ is of degree at least 3 and $v$ gives at most $4 \times 1 / 2=2$. We obtain $d^{*}(v) \geq 5-2=3$.

$d_{G}(v)=k \geq 6$.

In this case $v$ gives at most $k \times(1 / 2)$ so that $d^{*}(v) \geq k-k / 2=k / 2 \geq 6 / 2=3$.

Therefore, every vertex in $G$ gets a modernized degree of at least 3 and the theorem is proved.

Remark 9 The previous result also holds for graphs with maximum degree 2 and for graphs with maximum degree 3 (by the result from (6) but the question remains open for graphs with maximal degree 4 .

As previously, for planar graphs we obtain:

Corollary 10 Every planar graph $G$ of girth $g \geq 6$ with $\Delta(G) \geq 5$ admits a $(\Delta(G)+2,2)$-incidence coloring. Therefore, $\chi_{i}(G) \leq \Delta(G)+2$.

For graphs with maximum average degree less than $22 / 9$, we have:

Theorem 11 Every graph $G$ with $\operatorname{mad}(G)<22 / 9$ admits a $(\Delta(G)+2,2)$-incidence coloring. Therefore, $\chi_{i}(G) \leq \Delta(G)+2$.

Proof: It is enough to consider the case of graphs with maximum degree at most 4 , since for graphs with maximum degree at least 5 the theorem follows from Theorem 8 . Suppose that the theorem is false and let $G$ be a minimal counter-example (with respect to the number of vertices and edges). Observe first that we have $\Delta(G) \geq 3$ since otherwise we obtain by Theorem 1 that $\chi_{i}(G) \leq 2 \Delta(G) \leq \Delta(G)+2$ and every $(\Delta(G)+2)$-incidence coloring of $G$ is obviously a $(\Delta(G)+2,2)$-incidence coloring.

We first show that $G$ cannot contain any of the configurations depicted in Figure 3 .

(1) This case is similar to case 1 of Theorem 8 . 


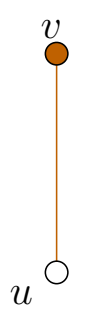

(1)

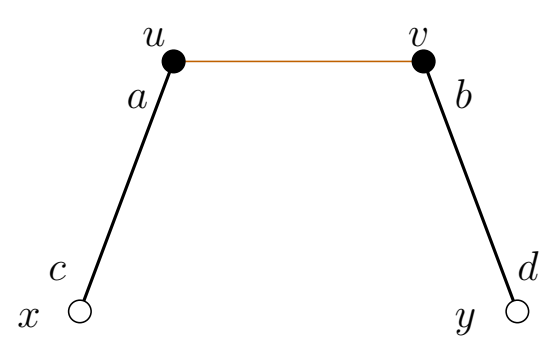

(2)

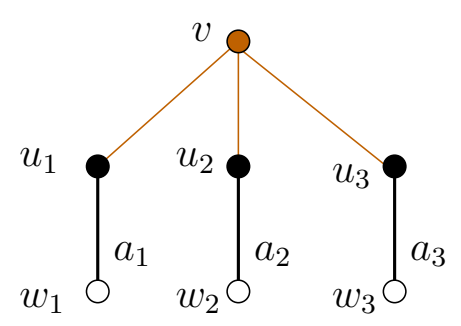

(3)

Fig. 3: Forbidden configurations for the proof of Theorem 11

(2) Let $x$ (resp. $y$ ) denote the other neighbor of $u$ (resp. $v$ ) in $G$. Due to the minimality of $G$, the graph $G^{\prime}=G \backslash u v$ admits a $(\Delta(G)+2,2)$-incidence coloring $\sigma^{\prime}$. We extend $\sigma^{\prime}$ to a $(\Delta(G)+2,2)$-incidence coloring $\sigma$ of $G$.

Suppose $\sigma^{\prime}(u, u x)=a, \sigma^{\prime}(v, v y)=b, \sigma^{\prime}(x, x u)=c$ and $\sigma^{\prime}(y, y v)=d$.

Suppose first that $|\{a, b, c, d\}|=4$. In that case, we set $\sigma(u, u v)=d$ and $\sigma(v, v u)=c$.

Now, if $|\{a, b, c, d\}| \leq 3$, we set $\sigma(u, u v)=e$ and $\sigma(v, v u)=f$ for any $e, f \notin\{a, b, c, d\}$.

(3) Let $u_{1}, u_{2}$ and $u_{3}$ denote the three neighbors of $v$ and $w_{i}$ denotes the other neighbor of $u_{i}, 1 \leq i \leq 3$, in $G$. Due to the minimality of $G$, the graph $G^{\prime}=G \backslash v$ admits a $(\Delta(G)+2,2)$-incidence coloring $\sigma^{\prime}$. We extend $\sigma^{\prime}$ to a $(\Delta(G)+2,2)$-incidence coloring $\sigma$ of $G$.

Suppose that $a_{i}=\sigma^{\prime}\left(w_{i}, w_{i} u_{i}\right), 1 \leq i \leq 3$. Since we have $\Delta(G)+2 \geq 5$ colors, there is a color $x$ distinct from $a_{i}$ for every $i, 1 \leq i \leq 3$.

Since $\left|F_{G}^{\sigma^{\prime}}\left(u_{i}, u_{i} w_{i}\right)\right|=\left|\sigma^{\prime}\left(I_{w_{i}}\right)\right| \leq \Delta(G)$ we have at least two colors for the incidence $\left(u_{i}, u_{i} w_{i}\right)$ for every $i, 1 \leq i \leq 3$. Thus, we can suppose $\sigma^{\prime}\left(u_{i}, u_{i} w_{i}\right) \neq x$ for every $i, 1 \leq i \leq 3$. We then set $\sigma\left(u_{i}, u_{i} v\right)=x$ for every $i, 1 \leq i \leq 3$.

Since $F_{G}^{\sigma}\left(v, v u_{i}\right)=\left\{x, \sigma^{\prime}\left(u_{i}, u_{i} w_{i}\right)\right\}$ for every $i, 1 \leq i \leq 3$, and since we have at least 5 colors, there are 3 distinct colors $c_{1}, c_{2}$ et $c_{3}$ such that $c_{i} \notin\left\{x, \sigma^{\prime}\left(u_{i}, u_{i} w_{i}\right)\right\}, 1 \leq i \leq 3$. We then set $\sigma\left(v, v u_{i}\right)=c_{i}$ for every $i, 1 \leq i \leq 3$.

Therefore, in all cases we obtain a $(\Delta(G)+2,2)$-incidence coloring of $G$, which contradicts our assumption.

We now associate with each vertex $v$ of $G$ an initial charge $d(v)=d_{G}(v)$, and we use the following discharging procedure: each vertex of degree at least 3 gives $2 / 9$ to each of its 2 -neighbors.

We shall prove that the modernized degree $d^{*}$ of each vertex of $G$ is at least $22 / 9$ which contradicts the assumption $\operatorname{mad}(G)<22 / 9$. Let $v$ be a vertex of $G$; we consider the possible cases for old degree $d_{G}(v)$ of $v$ (since $G$ does not contain the configuration $3(1)$, we have $d_{G}(v) \geq 2$ ):

$d_{G}(v)=2$.

Since $G$ does not contain the configuration $3(2)$ the two neighbors of $v$ are of degree at least 3 . Therefore, $v$ receives then $2 / 9$ from each of its neighbors so that $d^{*}(v)=2+2 / 9+2 / 9=22 / 9$. 


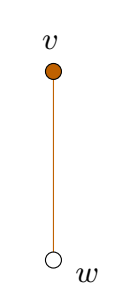

(1) $d_{G}(v)=1$

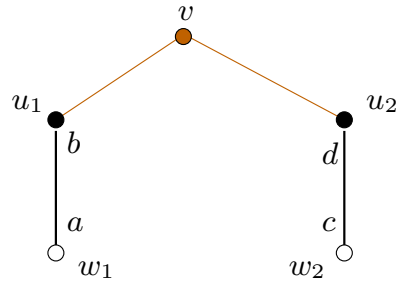

(2) $d_{G}\left(w_{1}\right)<\Delta(G)$

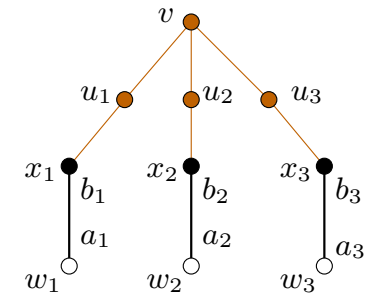

(3) $d_{G}\left(w_{2}\right)=d_{G}\left(w_{3}\right)=3$

Fig. 4: Forbidden configurations for the proof of Theorem 13

$d_{G}(v)=3$.

Since $G$ does not contain the configuration $3(3), v$ is adjacent to at most two 2 -vertices and $v$ gives at most $2 \times 2 / 9=4 / 9$. We obtain $d^{*}(v) \geq 3-4 / 9=23 / 9 \geq 22 / 9$.

$d_{G}(v)=4$.

In this case, $v$ gives at most $4 \times 2 / 9=8 / 9$ so that $d^{*}(v) \geq 4-8 / 9=28 / 9 \geq 22 / 9$.

Therefore, every vertex in $G$ gets a modernized degree of at least 3 and the theorem is proved.

By considering cycles of length $\ell \not \equiv 0 \quad(\bmod 3)$, we get that the upper bound of Theorem 11 is tight.

As previously, for planar graphs we obtain:

Corollary 12 Every planar graph $G$ of girth $g \geq 11$ admits $a(\Delta(G)+2,2)$-incidence coloring. Therefore, $\chi_{i}(G) \leq \Delta(G)+2$.

Finally, for graphs with maximum average degree less than 16/7, we have:

Theorem 13 Every graph $G$ with $\operatorname{mad}(G)<16 / 7$ and $\Delta(G) \geq 4$ admits $a(\Delta(G)+1,1)$-incidence coloring. Therefore, $\chi_{i}(G)=\Delta(G)+1$.

Proof: Since for every graph $G, \chi_{i}(G) \geq \Delta(G)+1$, it is enough to prove that $G$ admits a $(\Delta(G)+1,1)$ incidence coloring. Suppose that the theorem is false and let $G$ be a minimal counter-example (with respect to the number of vertices). We first show that $G$ cannot contain any of the configurations depicted in Figure 4

(1) This case is similar to case 1 of Theorem 8

(2) Let $u_{i}, i=1,2$, be the two neighbors of $v$ and $w_{i}$ denote the other neighbor of $u_{i}$ in $G$. Due to the minimality of $G$, the graph $G^{\prime}=G \backslash v$ admits a $(\Delta(G)+1,1)$-incidence coloring $\sigma^{\prime}$. We extend $\sigma^{\prime}$ to a $(\Delta(G)+1,1)$-incidence coloring $\sigma$ of $G$.

Suppose that $\sigma^{\prime}\left(w_{1}, w_{1} u_{1}\right)=a, \sigma^{\prime}\left(u_{1}, u_{1} w_{1}\right)=b, \sigma^{\prime}\left(w_{2}, w_{2} u_{2}\right)=c$ and $\sigma^{\prime}\left(u_{2}, u_{2} w_{2}\right)=d$. Since $\left|F_{G^{\prime}}^{\sigma^{\prime}}\left(w_{1}, w_{1} u_{1}\right) \cup\{c\}\right|=\left|\sigma^{\prime}\left(I_{w_{1}}\right) \backslash\{a\} \cup \sigma^{\prime}\left(A_{w_{1}}\right) \cup\{c\}\right| \leq \Delta(G)-2+1+1=\Delta(G)$, we can suppose that $a \neq c$. We then set $\sigma\left(v, v u_{1}\right)=a$ and $\sigma\left(v, v u_{2}\right)=c$.

Now, since $F_{G}^{\sigma}\left(u_{1}, u_{1} v\right) \cup F_{G}^{\sigma}\left(u_{2}, u_{2} v\right)=\{a, b, c, d\}$ and since we have at least $\Delta(G)+1 \geq 5$ colors, there is a color $x$ such that $x \notin\{a, b, c, d\}$. We then set $\sigma\left(u_{1}, u_{1} v\right)=\sigma\left(u_{2}, u_{2} v\right)=x$. 
(3) Let $u_{i}, 1 \leq i \leq 3$ be the three neighbors of $v, x_{i}$ denote the other neighbor of $u_{i}$ and $w_{i}$ denote the other neighbor of $x_{i}$ in $G$. Due to the minimality of $G$, the graph $G^{\prime}=G \backslash\left\{v, u_{1}, u_{2}, u_{3}\right\}$ admits a $(\Delta(G)+1,1)$-incidence coloring $\sigma^{\prime}$. We extend $\sigma^{\prime}$ to a $(\Delta(G)+1,1)$-incidence coloring $\sigma$ of $G$.

Suppose that $\sigma^{\prime}\left(w_{i}, w_{i} x_{i}\right)=a_{i}$ and $\sigma^{\prime}\left(x_{i}, x_{i} w_{i}\right)=b_{i}$ for every $i, 1 \leq i \leq 3$. Since $\mid F_{G}^{\sigma^{\prime}}\left(w_{i}, w_{i} x_{i}\right) \cup$ $\left\{b_{1}\right\}|=| \sigma^{\prime}\left(I_{w_{i}}\right) \backslash\left\{a_{i}\right\} \cup\left\{b_{i}, b_{1}\right\} \mid \leq 2+2=4$ for $i=2,3$, and since we have $\Delta(G)+1 \geq 5$ colors, we can suppose that $a_{2} \neq b_{1} \neq a_{3}$. We then set $\sigma\left(u_{i}, u_{i} x_{i}\right)=a_{i}$ and $\sigma\left(u_{i}, u_{i} v\right)=b_{1}$ for every $i$, $1 \leq i \leq 3$.

Since $F_{G}^{\sigma}\left(v, v u_{j}\right) \cup F_{G}^{\sigma}\left(x_{j}, x_{j} u_{j}\right)=\left\{b_{1}, b_{j}, a_{j}\right\}$ for $j=2,3$, there are two distinct colors $c_{2}$ and $c_{3}$ such that $c_{j} \notin\left\{b_{1}, b_{j}, a_{j}\right\}, j=2,3$. We set $\sigma\left(v, v u_{j}\right)=\sigma\left(x_{j}, x_{j} u_{j}\right)=c_{j}, j=2,3$.

Now, since $F_{G}^{\sigma^{\prime}}\left(v, v u_{1}\right) \cup F_{G}^{\sigma^{\prime}}\left(x_{1}, x_{1} u_{1}\right)=\left\{a_{1}, b_{1}, c_{2}, c_{3}\right\}$ and since we have at least 5 colors, there is a color $c_{1}$ such that $c_{1} \notin\left\{a_{1}, b_{1}, c_{2}, c_{3}\right\}$. We then set $\sigma\left(v, v u_{1}\right)=\sigma\left(x_{1}, x_{1} u_{1}\right)=c_{1}$.

Therefore, in all cases we obtain a $(\Delta(G)+1,1)$-incidence coloring of $G$, which contradicts our assumption.

We now associate with each vertex $v$ of $G$ an initial charge $d(v)=d_{G}(v)$, and we use the following discharging procedure:

(R1) each vertex of degree 3 gives $2 / 7$ to each of its 2-neighbors which has a 2-neighbor adjacent to a 3 -vertex and gives $1 / 7$ to its other 2-neighbors.

(R2) each vertex of degree at least 4 gives $2 / 7$ to each of its 2 -neighbors and gives $1 / 7$ to each 2 -vertex which is adjacent to one of its 2-neighbors.

We shall prove that the modernized degree $d^{*}$ of each vertex of $G$ is at least $16 / 7$ which contradicts the assumption $\operatorname{mad}(G)<16 / 7$. Let $v$ be a vertex of $G$, we consider the possible cases for old degree $d_{G}(v)$ of $v$ (since $G$ does not contain the configuration $4(1)$, we have $d_{G}(v) \geq 2$ ):

$d_{G}(v)=2$. In this case we consider five subcases:

1. $v$ has two 2-neighbors, say $z_{1}$ and $z_{2}$. Let $y_{i}$ be the other neighbor of $z_{i}, i=1,2$, in $G$. Since $G$ does not contain the configuration $4(2), y_{i}$ is of degree $\Delta(G) \geq 4$ for $i=1,2$. Each $y_{i}$, $i=1,2$, gives $1 / 7$ to $v$ so that $d^{*}(v)=2+1 / 7+1 / 7=16 / 7$.

2. $v$ is adjacent to a 3 -vertex $z_{1}$ and a 2 -vertex which is itself adjacent to a 3 -vertex. In this case $v$ receives $2 / 7$ from $z_{1}$ and we have $d^{*}(v)=2+2 / 7=16 / 7$.

3. $v$ is adjacent to a 3 -vertex $z_{1}$ and a 2 -vertex which is itself adjacent to a vertex $z_{2}$ of degree at least 4 . In this case $v$ receives $1 / 7$ from $z_{1}$ and $1 / 7$ from $z_{2}$ so that $d^{*}(v)=2+1 / 7+1 / 7=$ $16 / 7$.

4. $v$ is adjacent to two 3 -vertices that both gives $1 / 7$ to $v$ so that $d^{*}(v)=2+1 / 7+1 / 7=16 / 7$.

5. One of the two neighbors of $v$ is of degree at least 4 . In this case $v$ receives at least $2 / 7$ so that $d^{*}(v) \geq 2+2 / 7=16 / 7$.

$d_{G}(v)=3$.

Let $u_{1}, u_{2}$ and $u_{3}$ be the three neighbors of $v$. We consider two subcases according to the degrees of $u_{i}$ 's. 
1. One of the $u_{i}$ 's is of degree at least 3 , say $u_{1}$. In this case $v$ gives at most $2 / 7$ to $u_{2}$ and $2 / 7$ to $u_{3}$ so that $d^{*}(v) \geq 3-2 / 7-2 / 7=17 / 7 \geq 16 / 7$.

2. All the $u_{i}$ 's are of degree 2 . Let $x_{i}$ be the other neighbor of $u_{i}$ in $G, 1 \leq i \leq 3$.

(a) One of the $x_{i}$ 's is of degree at least 3 , say $x_{1}$. In this case $v$ gives $1 / 7$ to $u_{1}$, at most $2 / 7$ to $u_{2}$ and at most $2 / 7$ to $u_{3}$. We then have $d^{*}(v) \geq 3-1 / 7-2 / 7-2 / 7=16 / 7$.

(b) All the $x_{i}$ 's are of degree 2. Let $w_{i}$ be the other neighbor of $x_{i}$ in $G, 1 \leq i \leq 3$. Since $G$ does not contain the configuration $4(2)$ we have $d_{G}\left(w_{i}\right) \geq 3$ for every $i, 1 \leq i \leq 3$, and since $G$ does not contain the configuration $4(3)$, at most one of the $w_{i}$ 's, $1 \leq i \leq 3$, can be of degree 3 . Thus, we can suppose without loss of generality that $d_{G}\left(w_{1}\right)$ and $d_{G}\left(w_{2}\right) \geq 4$. In this case, $v$ gives $1 / 7$ to $w_{1}, 1 / 7$ to $w_{2}$ and at most $2 / 7$ to $w_{3}$. We then have $d^{*}(v) \geq 3-1 / 7-1 / 7-2 / 7=17 / 7 \geq 16 / 7$.

$d_{G}(v)=k \geq 4$.

In this case, $v$ gives at most $k \times(2 / 7+1 / 7)=3 k / 7$ so that $d^{*}(v) \geq k-3 k / 7=4 k / 7 \geq 16 / 7$.

Therefore, every vertex in $G$ gets a modernized degree of at least $16 / 7$ and the theorem is proved.

Considering the lower bound discussed in Section 1, we get that the upper bound of Theorem 13 is tight.

Remark 14 For every graph $G$, the square of $G$, denoted by $G^{2}$, is the graph obtained from $G$ by linking any two vertices at distance at most 2 . It is easy to observe that providing a $(k, 1)$-incidence coloring of $G$ is the same as providing a proper $k$-vertex-colouring of $G^{2}$, for every $k$ (by identifying for every vertex $v$ the color of $A_{v}$ in $G$ with the color of $v$ in $G^{2}$ ). By considering the cycle $C_{4}$ on 4 four vertices (note that $C_{4}^{2}=K_{4}$ ) we get that the previous result cannot be extended to the case $\Delta=2$. Consider now the graph $H$ obtained from the cycle $C_{5}$ on five vertices by adding one pending edge with a new vertex. Since $H^{2}$ contains a subgraph isomorphic to $K_{5}$, we similarly get that the previous result cannot be extended to the case $\Delta=3$.

As previously, for planar graphs we obtain:

Corollary 15 Every planar graph $G$ of girth $g \geq 16$ and with $\Delta(G) \geq 4$ admits a $(\Delta(G)+1,1)$ incidence coloring. Therefore, $\chi_{i}(G)=\Delta(G)+1$. 


\section{References}

[1] I. Algor and N. Alon, The star arboricity of graphs, Discrete Math. 75 (1989) 11-22.

[2] O.V. Borodin, A.V. Kostochka, J. Nešetřil, A. Raspaud and E. Sopena, On the maximum average degree and the oriented chromatic number of a graph, Discrete Math. 206 (1999) 77-89.

[3] R.A. Brualdi and J.J.Q. Massey, Incidence and strong edge colorings of graphs, Discrete Math. 122 (1993) 51-58.

[4] B. Guiduli, On incidence coloring and star arboricity of graphs, Discrete Math. 163 (1997) 275-278.

[5] M. Hosseini Dolama, E. Sopena and X. Zhu, Incidence coloring of $k$-degenerated graphs, Discrete Math. 283 (2004) 121-128.

[6] M. Maydanskiy, The incidence coloring conjecture for graphs of maximum degree 3, Discrete Math. 292 (2005) 131-141.

[7] W.C. Shiu, P.C.B. Lam and D.L. Chen, On incidence coloring for some cubic graphs, Discrete Math. 252 (2002) 259-266.

[8] S.D. Wang, D.L. Chen and S.C. Pang, The incidence coloring number of Halin graphs and outerplanar graphs, Discrete Math. 256 (2002) 397-405. 